\title{
Takotsubo Cardiomyopathy and Hip Fracture - Delay the Case or Proceed to Surgery?
}

\author{
Alexander Izakson ${ }^{* 1,3}$ and Irena Nordkin ${ }^{2,3}$ \\ ${ }^{1}$ Departments of Anesthesiology, Ziv Medical Centre, Israel \\ ${ }^{2}$ Department of Cardiology, Ziv medical Center, Israel \\ ${ }^{3}$ Azrieli Faculty of Medicine, Bar-Ilan University, Israel
}

Received: May 02, 2018; Published: May 16, 2018

*Corresponding author: Alexander Izakson, Department of Aneshesiology, Ziv Medical Center; Derech Ha Rambam, Safed, Israel

\begin{abstract}
Background: In most cases, Takotsubo cardiomyopathy is triggered by emotional or physical stress and is a unique form of reversible cardiomyopathy. Outcomes of the disease and possible complications are still unknown in patients who have to undergo surgery. We report a case of an elderly woman with a hip fracture and stress induced cardiomyopathy who underwent surgery shortly after the onset of the illness.
\end{abstract}

\section{Case Report}

A 75-year-old woman was admitted to our hospital with fracture of a left hip after she fell accidentally at home. The patient reported no loss of consciousness, dizziness or palpitation as the cause of the fall. On admission she complained of pain at the fracture site and pain in her chest of one hour's duration radiating to both shoulders. Her previous medical history included hypertension and hyperlipidemia without regular medical treatment, and a right hip replacement 6 months previously. In the emergency room she was treated with analgesics and antihypertensive medication. She was hemo dynamically stable. ECG showed normal sinus rhythm with mild ST-segment elevation in V2-V4. Troponin I was elevated at $0.8 \mathrm{ng} / \mathrm{ml}$. With strong suspicion of acute coronary syndrome the patient was admitted to the Coronary Care Unit for emergency cardiac catheterization. Coronary angiography was normal and left ventriculography demonstrated apical ballooning with preserved basal left ventricular contraction. Stress induced cardiomyopathy was diagnosed and therapy for this condition started.

The decision of the multidisciplinary consultation between the cardiologist, orthopedist and anesthetist was to operate on the patient's fractured femur. The patient underwent left bipolar hip endoprosthesis within 48 hours of hospital admission. Surgery was uneventful and performed under general anesthesia. During hospitalization the patient remained stable. After discharge she was transferred to a rehabilitation facility. On follow-up two months later the patient was asymptomatic. Echocardiography examination showed normal left ventricular ejection fraction without wall motion abnormalities, a finding consistent with complete recovery of myocardial function, typical for this form of cardiomyopathy.

\section{Discussion}

Takotsubo or stress cardiomyopathy is a form of heart failure generally characterized by acute onset of transient systolic dysfunction of the apical and/or mid segments of the left ventricle. Clinical presentation and ECG findings may mimic ST-segment elevation myocardial infarction, even in the absence of obstructive coronary artery disease [1]. This case deals with the problem of urgent surgery in the patient with Takotsubo cardiomyopathy. There is limited data about the outcome and complications of patients operated during the onset of this illness. We aware of only 4 cases of Takotsubo cardiomyopathy in elderly females after hip fracture. In all cases uneventful hip surgery was performed within an average period of six days after almost complete normalization of left ventricular function [2]. There are real benefits of early surgery in patients with hip fractures in terms of respiratory and cardiovascular complications [3]. However, as well as causing distress to the patient, delay in surgery greater than 48 hours is associated with prolonged length of hospital stay, later mobilization and a greater incidence of complications, especially respiratory, and the development of pressure sores as well as reduced chance of successful internal fixation and rehabilitation [4]. 
The fact that our patient was in a stable hemodynamic condition without obvious signs of heart failure greatly influenced our decision to proceed with surgery, After a comprehensive discussion with the members of the operating team and evaluation of possible benefits and risks to the patient, we decided to proceed with the surgery under general endotracheal anesthesia. Our understanding was that in this specific case general anesthesia might provide more hemodynamic stability for this patient and preclude the development of further cardiovascular deterioration [5]. Despite reduced left ventricular function our patient tolerated the procedure exceptionally well and enjoyed the benefits of timely performed hip fracture surgery. Our case is a unique one where we describe the case of a patient with fractured hip who underwent surgery within 48 hours on the onset of Takotsubo cardiomyopathy.

\section{Conclusion}

As seen in our case, a hemo-dynamically stable patient with hip fracture and acute stress cardiomyopathy may have surgery under general anesthesia before left ventricular function becomes normalized.

\section{Reference}

1. Prasad A, Lerman A, Rihal CS (2008) apical ballooning syndrome (TakoTsubo or stress cardiomyopathy): a mimic of acute myocardial infarction. Am Heart J 155(3): 408.

2. Rostagno C, Cammilli A, Di Cristo A, Polidori G, Cartei A, et al. (2015) Takotsubo cardiomyopathy in four elderly females after hip fracture. Research 2: 1321-1325.

3. Smektala R, Endres HG, Dasch B, Maier C, Trampisch HJ, et al. (2008) The effect of time-to-surgery on outcome in elderly patients with proximal femoral fractures. BMC Musculoscelet Disord 9: 171.

4. Vidal EI, Moreira Filho DC, Coeli CM, Camargo KR, Fukushima FB, et al. (2009) Hip fracture in the elderly: does counting time from fracture to surgery or from hospital admission to surgery matter when studying inhospital mortality? Osteoporos Int 20(5): 723-729.

5. Luger T, Kammerlander C, Gosch M, Luger MF, Kammerlander-Knauer U, et al. (2010) Neuroaxial versus general anaesthesia in geriatric patients for hip fracture surgery: does it matter? Osteoporos Int 21(suppl 4): S555e72.
This work is licensed under Creative Commons Attribution 4.0 License

Submission Link: https://biomedres.us/submit-manuscript.php

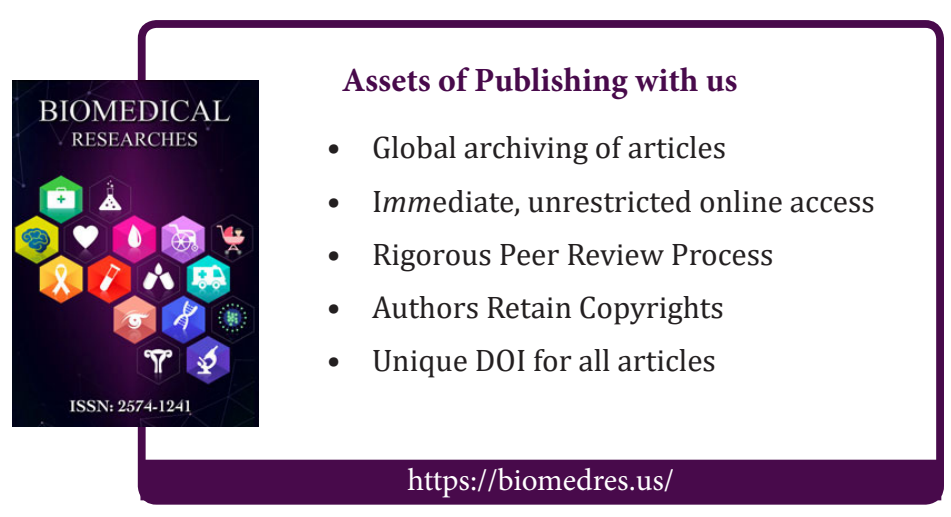

